\title{
Les conceptions d'enseignants de 31 pays sur la santé, entre approches biomédicale et socio-écologique
}

\author{
Pierre Clément \\ Université d'Aix-Marseille, EA 4671-ADEF, Gestepro, France \\ clement.grave@free.fr
}

\author{
Graça S. Carvalho \\ CIEC, Universidade do Minho, Braga, Portugal \\ graca@ie.uminho.pt
}

RÉSUMÉ • La définition de la santé a longtemps été limitée à l'approche biomédicale, comme "une absence de maladie ou d'infirmité". Elle s'est ensuite élargie pour devenir moins négative, plus holistique, intégrant les paramètres individuels, sociaux et écologiques qui permettent d'être en bonne santé et de l'améliorer (Promotion de la santé). Le présent travail analyse les réponses de 11312 enseignants de 31 pays à une question leur permettant de situer la santé en fonction de ces deux pôles, biomédical et socio-écologique. Les réponses de la grande majorité des enseignants ne se limitent pas à l'un des deux pôles, et associent les deux. Mais il existe de fortes différences entre pays, qui correspondent aux tendances, plus tranchées encore, mises en évidence par des travaux antérieurs sur l'analyse des manuels scolaires de biologie dans plusieurs de ces pays. Les enseignants français, traditionnellement attachés à une conception biomédicale, commencent à s'ouvrir à une approche plus socio-écologique de la santé.

MOTS-CLÉS • santé, éducation, conceptions, enseignants du primaire, enseignants du secondaire, Europe, Afrique, Asie, Australie, Brésil

Nous tenons à remercier Charline Laurent, qui a effectué les analyses statistiques pour ce travail, ainsi que toutes les personnes qui, dans chacun des 31 pays, ont recueilli les données. Ne sont cités ici que les responsables des équipes de chaque pays : (1) soit dans le cadre du projet de recherche BIOHEAD-Citizen : Algérie : Farida Khammar; Allemagne : Franz Bogner; Chypre: Nicos Valanides; Estonie : Kai Pata \& Tago Sarapuu; Finlande : Anna-Liisa Rauma-Kosonen; France : Pierre Clément \& Daniel Favre; Grande-Bretagne: Stephen Tomkins; Hongrie : Attila Varga ; Italie : Silvia Caravita \& Adriana Valente; Liban : Iman Khalil; Lituanie : Jurga Turcinaviciene; Malte : Paul Pace; Maroc: Sabah Selmaoui; Pologne : Elwira SamonekMiciuk; Portugal : Graça Carvalho; Roumanie : Adrienne Kozan-Naumescu; Sénégal : Mame Seyni Thiaw; Tunisie : Mondher Abrougui. (2) soit ultérieurement : Australie : Frances Quinn; Bénin : Thierry Dovonou; Brésil : Graziela Lopez \& Paloma Silva; Burkina Faso : Ivette Béré - Yoda ; Cameroun : Lawrence Ntam 
Nchia; Gabon : Laurence Ndong; Russie : Frédéric Charles; Serbie : Jelena Stanisavljevic; Afrique du Sud: Michele Stears; Espagne : José María Sanchis; Corée du Sud : Hae-Ae Seo; Suède : Niklas Gericke; Togo : Mensan Azadzi Dzamayovo.

Le présent article a aussi bénéficié d'une partie du financement du projet Stratégique UID/ CED/00317/2013 de la FCT et du CIEC (Centre de Recherche sur les Études de l'Enfant) au Portugal.

\section{Introduction : deux modèles d'éducation à la santé}

Alors qu'actuellement prévaut une conception holistique de la santé, pendant très longtemps le monde occidental a réduit la santé à sa dimension biomédicale, centrée sur les aspects biologiques et médicaux des maladies, avec plus récemment une ouverture sur leur prévention. Ce modèle biomédical est apparu au XVIII ${ }^{\mathrm{e}}$ siècle en Europe occidentale, le siècle des Lumières, avec l'hégémonie du rationalisme et de la science, supplantant la religion qui, auparavant, déterminait la compréhension du monde et en particulier la perception de la maladie et de la santé (Naidoo \& Wills, 1994). Le temps du rationalisme se caractérise, selon Foucault (1975), par une domination quasi despotique de la science et de la technologie qui contrôlent de plus en plus, non seulement les forces productives, mais aussi les décisions politiques, conduisant à un rationalisme d'État qui impose des formes de gouvernance et des processus de contrôle, mais aussi qui détermine des mesures sociales normatives dont il ne faut pas s'écarter. C'est ainsi que commence à s'établir la notion de "normal" (versus "anormal") auquel on attribue aussi une valeur morale : "le normal correspond au bien" (versus "l'anormal correspondant au mal") (Canguilhem, 1979).

Le modèle biomédical de la santé est progressivement devenu dominant dans les conceptions sur la santé individuelle et collective. Le développement de la science et de la technologie a largement renouvelé à la fois nos connaissances dans le domaine biologique et la sophistication technologique dans le domaine médical, avec des succès incontestables dans le domaine médical et chirurgical. Cette valorisation de la méthode scientifique et de ses applications s'est focalisée sur le corps humain, objet d'étude central. Tout ce qu'on pouvait mesurer et cataloguer était reconnu comme "réel" dans un sens objectif et universel. La conception de la santé comme absence de maladie s'est structurée dans le monde médical où la maladie est considérée comme un état pathologique identifiable : un écart par rapport aux paramètres normaux mesurables dans un corps sain ; ces écarts peuvent être diagnostiqués et classés (Katz, Peberdy \& Douglas, 2000). L'idée de base est que le corps fonctionne comme une machine : le modèle biomédical est ainsi basé sur les conceptions suivantes (Carvalho, 2012, p. 37) :

"(i) toutes les parties du corps sont reliées, mais peuvent être isolées et traitées séparément; (ii)

être en bonne santé signifie avoir toutes les parties du corps en bon état de fonctionnement; (iii) être malade consiste à avoir des déficiences dans des parties du corps; (iv) la maladie est causée 
par des processus internes (par exemple la dégénérescence liée à l'âge, le non fonctionnement de l'auto-régulation) ou par des processus externes (comme l'invasion par des microorganismes pathogènes); ( v) le traitement médical a pour but de restaurer les fonctions normales du corps, sa santé".

En bref, le modèle biomédical se concentre explicitement sur les causes, le traitement et la prévention des maladies. Les professionnels de santé sont reconnus comme les personnes disposant des connaissances et des compétences pour identifier la maladie et ses causes, ainsi que pour la traiter, et donc jouent un rôle prépondérant, tout en utilisant souvent des méthodes de persuasion paternalistes pendant leur travail (Ewles \& Simnett, 1999). Selon ce modèle, ce sont des professionnels de la santé qui ont la responsabilité de veiller à ce que les patients se conforment aux prescriptions médicales et d'encourager l'utilisation de procédures qui sont censées être capables de prévenir et de réduire les maladies (Carvalho, 2006).

D'une façon tout à fait opposée au modèle dominant biomédical de la santé, Antonovsky (1996) s'est intéressé non pas aux causes qui provoquent la maladie, mais plutôt à celles qui entraînent une bonne santé : l'approche salutogénique. Dans ce cadre conceptuel, l'accent est mis sur ce qui permet aux personnes d'être en bonne santé et de l'améliorer, en considérant que les facteurs de stress et les perturbations peuvent être évités : ainsi la relation dynamique entre l'individu et son environnement devient extrêmement importante. En ce sens, il est essentiel que les personnes créent elles-mêmes leurs propres ressources en acquérant les compétences nécessaires pour lutter en permanence contre les agents de perturbation. Elles créent ainsi un "sens de la cohérence " qui nécessite l'intégration des trois éléments suivants (Antonovsky, 1979) :

(i) la compréhensibilité : les stimuli déclenchés par l'environnement interne ou externe tout au long de la vie sont structurés et peuvent être prévus et expliqués; (ii) la combativité : chaque personne a des ressources disponibles pour répondre aux besoins créés par ces stimuli; (iii) l'importance : ces besoins sont des défis dignes d'investissement et de prise en compte.

La combativité dépend non seulement des ressources propres à chaque personne, mais aussi de ses relations avec d'autres et du soutien social et environnemental. Donc ce cadre "salutogène" donne une grande importance à la fois aux personnes et à leur contexte social et environnemental.

Il établit ainsi un pont entre l'approche biomédicale et l'approche socio-écologique qui, au lieu de ne traiter de la santé que comme "une absence de maladie ou d'infirmité" (OMS, 1946), est basée sur une attitude positive et holistique de la santé, en donnant une grande importance à l'éducation et à la promotion de la santé, car chaque personne peut se transformer, se former (Martins, El-Hani \& Carvalho, 2016). L'approche socio-écologique privilégie l'empowerment de toute personne en tant qu'agent actif de sa santé individuelle et collective, ce qui est à la base de la promotion de la santé (Martins, El-Hani \& Carvalho, 2016). Le concept de "Promotion de la santé" a été formulé par l'Organisation mondiale de la santé (OMS, 1986, p. 1) : 
Il a pour " but de donner aux individus davantage de maîtrise de leur propre santé et davantage de moyens de l'améliorer [... . . L'individu, ou le groupe, doit pouvoir identifier et réaliser ses ambitions, satisfaire ses besoins et évoluer avec son milieu ou s'y adapter. La santé est donc perçue comme une ressource de la vie quotidienne, et non comme le but de la vie; c'est un concept positif mettant l'accent sur les ressources sociales et personnelles, et sur les capacités physiques".

Ainsi la santé peut être comprise de différentes façons, avec un continuum entre deux pôles : d'un côté le modèle biomédical (BM), qui considère la santé uniquement comme absence de maladie donc centrée sur les soins et la guérison, et à l'autre pôle le modèle socio-écologique (SE), où la santé correspond à une attitude active et positive plus globale, de promotion de la santé par l'amélioration de soi et du contexte social et environnemental de chacun. Le premier pôle, BM, concerne l'anatomo-pathologie, le traitement curatif et la prévention relative à telle ou telle maladie, tandis que le second, SE, concerne tous les modes de vie qui permettent d'être en bonne santé, ce que les Anglais nomment "empowerment", et concerne aussi les contextes sociaux et environnementaux qui permettent à chaque personne de s'épanouir, d'être en bonne santé. L'absence de pollution de l'air ou de l'eau, ou encore la qualité des conditions de travail, deviennent alors des préoccupations majeures.

Jusqu'à quel point l'Éducation à la santé s'intéresse-t-elle à ces deux modèles de la santé, ou privilégie-t-elle l'un des deux? C'était un des thèmes du projet de recherche européen BIOHEAD-Citizen (Biology, Health and Environmental Education for better Citizenship : Carvalho, Clément et al., 2008). Il concernait, pour l'Éducation à la santé, d'une part l'analyse des programmes et manuels scolaires des 19 pays impliqués (13 en Europe, 5 en Afrique et 1 au Moyen-Orient : Carvalho, 2008; Carvalho, Dantas et al., 2008), d'autre part l'analyse des conceptions d'enseignants dans ces mêmes pays (Carvalho, 2008; Berger et al., 2011; Jourdan et al., 2013). Ce second axe de recherche a ensuite été prolongé dans de nouveaux pays, sous la responsabilité de P. Clément, et nous présentons ici des résultats relatifs à 31 pays. Nous n'avons, jusqu'à présent, publié que les résultats relatifs au thème de l'évolution dans 30 de ces pays (Clément, 2014a), ou encore ceux relatifs au thème du déterminisme génétique de traits humains dans 23 de ces pays (Castéra \& Clément, 2014). Les publications des résultats relatifs à l'éducation à la santé (Carvalho, 2008; Berger et al., 2011; Jourdan et al., 2013) n'ont, jusqu'ici, porté que sur 15 ou 16 pays et leurs analyses étaient plus qu'incomplètes, plusieurs des effets indiqués comme significatifs (par exemple l'effet religion, ou encore l'effet sexe) n'étant, comme nous le montrons dans le présent travail, que des conséquences des différences entre pays. Donc non seulement les résultats présentés ici sont totalement nouveaux pour 15 des 31 pays (Afrique du Sud, Algérie, Australie, Bénin, Brésil, Burkina Faso, Cameroun, Corée du Sud, Espagne, Gabon, Grande-Bretagne, Russie, Serbie, Suède, Togo), mais, de plus, les analyses des données issues des 31 pays sont originales, y compris les données déjà publiées pour 16 des pays du projet BIOHEAD-Citizen. 
L'ensemble de ce projet a tenté d'analyser les conceptions des enseignants en tant que possibles interactions KVP entre leurs connaissances scientifiques (K pour knowledge), leurs valeurs (V) et leurs pratiques sociales (P) (Clément, 2004, 2010, 2016). II n'est pas besoin de revenir ici sur les connaissances relatives aux aspects biomédicaux (BM) de la santé : elles marquent des succès croissants, souvent spectaculaires, pour guérir de certaines maladies, ou pour développer des politiques de prévention, jusqu'à l'éradication de certaines endémies. Mais ces succès sont aussi la conséquence de l'amélioration des conditions de vie. C'est particulièrement évident dans les pays du Tiers-Monde. Claire Brisset notait déjà en 1984 que " 2 milliards d'hommes sont privés d'accès à l'eau potable " et que "le quart de la population du tiers monde à économie de marché souffre de malnutrition". La situation de ces pays ne s'est que partiellement améliorée depuis, et s'est même fortement dégradée dans ceux d'entre eux touchés par la guerre. Les analyses scientifiques des origines multiples de la malnutrition se sont développées, en particulier depuis l'ouvrage de Susan George (1978) qui montrait les effets désastreux de la révolution verte et de l'emprise de trusts internationaux sur l'agriculture et l'alimentation. Ces connaissances ne sont qu'une partie de celles sur lesquelles s'appuie l'OMS pour la promotion du modèle socio-écologique (SE) de la santé. Ainsi, des connaissances (K) fondent ces deux modèles de la santé (BM et SE). Elles sont complémentaires et s'appuient sur différentes "valeurs de la santé" (V), termes utilisés par Claudine Herzlich (1995), valeurs qui elles-mêmes correspondent à des pratiques sociales $(\mathrm{P})$ différentes et complémentaires : dans une perspective médicale, pratiques de guérison et de prévention pour le pôle BM, et des pratiques plus politiques, économiques et sociales pour la facette santé environnementale du pôle SE, et plus psycho-sociales pour la facette "empowerment". Sans même parler du tiers-monde, ces deux modèles de santé (BM et SE) concernent directement aussi les pays développés où les aspects environnementaux sont de plus en plus pris en compte : qualité de l'air, retraitement des eaux, pollutions par les pesticides, pour ne citer que quelques exemples; et où les conséquences des modes de travail sur la santé sont aujourd'hui soulignées : pénibilité de certaines tâches, stress des cadres, etc. Au total, les interactions KVP peuvent aider à caractériser les conceptions sur la santé, en mettant en lumière la complexité de toute analyse et action dans ce domaine, un peu comme les interactions KABP (Knowledge, Attitudes, Beliefs and Practices) ont été utilisées dans les enquêtes sur le SIDA pour montrer que l'information (K), même bien faite, ne suffit pas à induire des changements de comportements sexuels (Herzlich, 1992; Moatti et al., 1990).

Les conceptions de la santé varient fortement en fonction du contexte social. Herzlich (1992) les a étudiées, et rapporte par exemple les propos d'habitants d'un bidonville brésilien : "Je suis en bonne santé, puisque je vis", ce qui ne les empêche pas d'énumérer ensuite leurs maux et maladies. L'éducation à la santé varie aussi d'un pays à l'autre. L'ouvrage coordonné par Taylor et al. (2012) concerne 25 pays, consacrant un chapitre par pays, dont sept recoupent l'échantillon du présent travail : Liban (Kanj, 2012; Portugal (Carvalho, 2012); Hongrie (Chrappan, 2012); 
Russie (Gvozdeva \& Kirilina, 2012); Pologne (Platje \& Słodczyk, 2012); Afrique du Sud (Conradie, 2012); Malaisie (Yahya \& Ali, 2012). Des informations sur d'autres pays européens, en particulier la France, peuvent être consultées dans l'article de Carvalho et Berger (2012) et dans l'ouvrage de Pommier et Jourdan (2012). Ces derniers auteurs ont aussi mis au point diverses méthodes d'évaluation de l'implémentation de la promotion de la santé dans des écoles françaises (Pommier, Guével \& Jourdan, 2010). L'enjeu de ces recherches en France, pays où le modèle biomédical a été et reste dominant, est d'accompagner et d'évaluer la prise en compte par le système scolaire des préconisations de l'OMS dans sa Charte d'Ottawa (1992) : le modèle holistique, socio-écologique (SE) de promotion de la santé, intégrant une vision positive de la santé, du bien-être, qui ne se limite pas aux seules maladies.

II n'est pas possible ici de présenter la situation de l'éducation à la santé dans chacun des 31 pays avec lesquels nous avons travaillé, ni même de lister toutes les recherches réalisées jusqu'à présent sur l'éducation à la santé dans chacun de ces pays : les références indiquées plus haut sont loin d'être exhaustives. En effet, chaque situation locale ne peut être résumée en une ou deux phrases. Par exemple, la thèse de Fuddah (2014) a porté sur l'éducation à la santé dans des écoles au Liban, soit des écoles publiques libanaises, soit des écoles dans des camps palestiniens : la situation sanitaire est assez différente dans les deux cas, mais les conceptions des élèves, analysées par leurs participations à des "focus groups "', ne diffèrent guère (Fuddah, Khalil \& Clément, 2015). Dans la plupart des autres pays, la majorité des recherches porte sur l'implémentation de l'éducation à la santé en tant que discipline spécifique, ou au sein d'autres disciplines scolaires, ainsi que sur l'intérêt de faire intervenir dans les écoles d'autres acteurs que les enseignants, comme des spécialistes de la médecine. Mais un nombre croissant de recherches concerne aussi les défis du lien entre éducation à la santé et éducation à l'environnement et au développement durable (Zeyer \& Kyburz-Graber, 2012).

Le présent travail est uniquement focalisé sur les conceptions des enseignants relatives aux deux modèles de santé que nous venons de présenter (BM et SE) : jusqu'à quel point, malgré la diversité de leur contexte, les enseignants se sont-ils approprié les préconisations de l'OMS (1992)? Est-il possible de mettre en évidence des corrélations entre les conceptions de ces enseignants et certains des paramètres que nous pouvons contrôler, soit individuels (sexe, âge, formation, religion, etc.), soit collectifs (caractéristiques de leurs pays respectifs)?

\section{Hypothèses et questions de recherche}

Notre hypothèse principale, qui s'appuie sur les résultats obtenus pour d'autres thèmes du projet de recherche BIOHEAD-Citizen, tels que l'évolution (Clément, 2014a) ou la

1 On parle de "focus group" quand un groupe est constitué pour débattre d'un thème précis. 
génétique humaine (Castéra \& Clément, 2014), est qu'il y aura de fortes différences d'un pays à un autre. Nous essaierons alors de voir s'il est possible d'identifier des tendances dans ces différences, par exemple entre pays plus ou moins développés sur le plan économique, ou entre pays de différents continents ou situations climatiques.

Une facette des différences observées, indépendante ou non des différences entre pays, est relative à l'influence possible de la religion. En effet, Faure (1997) a noté que la notion de santé collective est née dans des contextes fortement religieux en Occident, à partir du XVle siècle, avec des différences qui "opposent les pays marqués par la Réforme, où les mesures de santé s'adressent à une population pour laquelle le corps est objet d'éducation, et les pays de tradition catholique, où la santé est plus l'affaire des autorités et des médecins que le problème de chacun" (Faure, 1997, p. 283). Nos résultats issus de l'analyse des textes et images de manuels scolaires (figures 7 et 8 ; Carvalho, Dantas et al., 2008) pourraient exprimer ce type de différences, l'Allemagne et la Finlande, de culture protestante, se situant vers le pôle "socio-écologique" (SE) tandis que la Pologne, la France, Malte et l'Italie, de culture catholique, se situent vers le pôle biomédical (BM). Nous analyserons donc si les conceptions des enseignants varient en fonction de leur propre religion.

Enfin, le modèle SE comportant des dimensions personnelles telles que l'estime de soi, ou se sentir en paix avec soi-même, il est possible que certains paramètres individuels, comme le sexe ou le type de formation universitaire, soient corrélés avec les conceptions analysées. Nous testerons l'existence de ces éventuelles corrélations.

Notre principale question de recherche est donc d'identifier comment, dans chacun des 31 pays avec lesquels nous avons travaillé, les conceptions des enseignants interrogés se situent entre les deux pôles que nous venons de définir : biomédical (BM) et socio-écologique (SE).

Nous compléterons cette comparaison entre pays par l'identification de variations des conceptions de ces enseignants en fonction d'autres paramètres contrôlés : leur religion tout particulièrement, en fonction de la seconde hypothèse formulée ci-dessus, mais aussi leur sexe, leur niveau de formation universitaire, leur statut (enseignants du primaire ou du secondaire, et pour ces derniers, enseignant la biologie ou la langue du pays).

Les différences liées à ces paramètres pouvant n'être que des conséquences des différences entre pays, par exemple plus ou moins d'hommes dans chaque pays parmi les enseignants interrogés, nous identifierons les effets qui persistent après avoir supprimé statistiquement l'effet pays.

Enfin, dans la discussion, nous mettrons en parallèle, dans les cas où ce sera possible, les conceptions des enseignants ( 31 pays) et le contenu des manuels scolaires analysés sur ce thème de l'éducation à la santé au cours du projet BIOHEAD-Citizen (18 de ces 31 pays: Carvalho, 2008; Carvalho, Dantas et al., 2008). 


\section{Méthodologie}

Dans ces 31 pays, les enseignants ont répondu, par écrit et de façon totalement anonyme, au questionnaire créé et validé par le projet de recherche BIOHEAD-Citizen (Carvalho, Clément et al., 2008). Les enseignants étaient toujours en groupe, dans l'établissement supérieur où ils terminaient leur formation pour les futurs enseignants, ou dans leur établissement scolaire pour les enseignants en service, en présence du chercheur qui ramassait immédiatement les questionnaires remplis afin qu'il n'y ait pas de non retour et pour garantir l'anonymat des réponses.

Le questionnaire comporte 155 questions fermées portant sur cinq thèmes : l'évolution, la génétique humaine (incluant les différences liées au sexe ou au groupe social), l'éducation à l'environnement, à la santé et à la sexualité. L'ordre des questions est aléatoire, sauf les 17 questions sur les caractéristiques personnelles de l'enseignant interrogé, situées dans une dernière partie.

Le questionnaire a été élaboré collectivement par les 19 équipes impliquées dans le projet de recherche BIOHEAD-Citizen, puis testé et validé durant les deux premières années du projet : questionnaire pilote beaucoup plus long, testé dans plusieurs pays, entretiens, stabilité des réponses du même public interrogé à un mois d'intervalle dans quelques pays, sélection des questions les plus fiables et les plus discriminantes, puis choix des analyses multivariées les plus pertinentes avec le logiciel libre " $R$ " (Clément \& Carvalho, 2007). Plusieurs questions portent sur l'éducation à la santé, dont une, la question A63, correspond précisément à notre question de recherche. Cette question est située au milieu du questionnaire.

En plus des réponses aux questions personnelles permettant de caractériser chaque enseignant (en particulier sa religion déclarée, mais aussi son âge, son sexe, son degré d'instruction, ses opinions politiques ou religieuses, etc.), nous n'utilisons ici que les réponses à cette question $\mathrm{A} 63$.

"A63. La santé peut être perçue de différentes manières. Dans la liste suivante, cocher les trois expressions qui sont les plus fortement associées à votre propre conception de la santé :

- Ne pas souffrir de maladies graves;

- Être en paix avec moi-même;

- Profiter de la vie, sans trop de stress;

- Avoir toutes les parties de mon corps qui fonctionnent bien;

- Être en forme pour être socialement actif ;

- Ne pas avoir besoin d'aller chez le docteur pour être soigné."

Trois des six items proposés (le premier, le quatrième et le sixième) expriment une conception biomédicale de la santé, tandis que les trois autres expriment une conception socio-écologique caractéristique de la promotion de la santé. Dans chaque questionnaire rempli, nous avons compté combien d'items relatifs au modèle socio-écologique ont été cochés : quatre possibilités, de zéro à trois (figure 1). 
Dans chaque pays, les enseignants interrogés relevaient de six catégories, avec si possible autour d'une cinquantaine d'enseignants pour chacune :

- InP : enseignants du primaire en service;

- PreP : enseignants du primaire en fin de formation;

- InB : enseignants du secondaire, biologie, en service;

- PreB : enseignants du secondaire, biologie, en fin de formation;

- InL : enseignants du secondaire, langue du pays, en service;

- PreL : enseignants du secondaire, langue du pays, en fin de formation.

Dans certains pays, comme les plus petits, l'échantillon est plus réduit. Dans d'autres il est plus important pour tester quelques hypothèses complémentaires. Ces échantillons n'ont pas la prétention d'être significativement représentatifs de l'ensemble de la population concernée dans chaque pays, même si la consigne a été de tenir compte au mieux de la diversité de cette population dans chaque pays : par exemple en interrogeant des professeurs d'école dans des zones urbaines, péri-urbaines et rurales : ou encore, au Liban, en tenant grand compte des proportions des différentes confessions. Au total, chaque échantillon n'est ainsi pas loin d'être représentatif. Ainsi, en France, où nous avons comparé deux régions (LanguedocRoussillon et Rhône-Alpes) sans trouver de différence entre les deux; les données ont été groupées. Dans les pays les plus grands, les données ont volontairement été recueillies dans une seule région (par exemple la Bavière en Allemagne; Oryol en Russie, ville de 500000 habitants située à $400 \mathrm{~km}$ au sud de Moscou) de façon à pouvoir par la suite la comparer à d'autres régions. Par simplification, certes abusive tant que la comparaison avec d'autres régions n'a pas été réalisée, nous avons conservé le nom du pays pour désigner ces échantillons.

Au total, 11312 enseignants ont été interrogés dans les 31 pays suivants :

Afrique du Sud (336), Algérie (223), Allemagne (365), Australie (201), Bénin (229), Brésil (402), Burkina Faso (296), Cameroun (523), Chypre (322), Corée du Sud (306), Espagne (318), Estonie (182), Finlande (306), France (732), Gabon (269), GrandeBretagne (154), Hongrie (334), Italie (559), Liban (848), Lituanie (316), Malte (198), Maroc (518), Pologne (311), Portugal (350), Roumanie (273), Russie (405), Sénégal (324), Serbie (314), Suède (377), Togo (270), Tunisie (753).

Dans chaque pays, le chercheur ou son équipe a renseigné un tableau Excel à partir des questionnaires remplis dans ce pays. Ces tableaux ont ensuite été centralisés en France pour des analyses statistiques réalisées par des statisticiens. Pour le présent travail, ces analyses ont été effectuées par Charline Laurent, en utilisant le logiciel "R" qui est en libre accès, en particulier sa partie "ADE 4" (R Development Core Team, 2006). 


\section{Résultats}

\subsection{Différences entre pays}

Dans la figure 1, les réponses à la question A63 ont été regroupées par pays, et les pays ont été ordonnés en fonction d'une majorité d'items biomédicaux cochés (3 ou 2 des 3 items biomédicaux, et donc 0 ou 1 item socio-écologique coché : couleurs noir et gris sombre dans la figure 1).

Cette figure 1 montre que, dans chaque pays, les enseignants interrogés n'ont pas tout à fait la même conception. Globalement, sur l'ensemble des personnes interrogées, il y a à peu près autant d'enseignants qui ont privilégié les items biomédicaux (BM) que d'enseignants qui ont privilégié les items socio-écologiques (SE). Dans chaque pays, les enseignants qui n'ont coché que des items biomédicaux sont peu nombreux (entre $0 \%$ et $15 \%$ ). Il en est de même pour les enseignants qui ont coché les 3 items socio-écologiques : entre $0 \%$ et $15 \%$, sauf au Maroc (41\%), en Finlande (23\%) et en Suède (21\%). Dans chaque pays, la majorité des enseignants a coché 1 item d'un modèle (BM ou SE) et 2 items de l'autre.

Les différences entre pays sont cependant très significatives $(p<0,0001)$.

Dans certains pays, les conceptions biomédicales de la santé dominent : $90 \%$ des enseignants ont coché 2 ou 3 items BM en Tunisie, $80 \%$ au Bénin, $71 \%$ en Roumanie, $66 \%$ en France, $65 \%$ en Allemagne, $62 \%$ en Pologne et $60 \%$ au Sénégal. Alors que ces proportions sont bien plus faibles dans d'autres pays : $30 \%$ en Suède, $32 \%$ en Corée du Sud, 34 \% en Lituanie ainsi qu'en Finlande, 35 \% au Maroc, 37 \% en Russie et $38 \%$ au Brésil : dans ces derniers pays, les conceptions socio-écologiques sont largement dominantes.

Le contexte de chaque pays (social, historique, économique, etc.) influence donc fortement les conceptions de la santé, mais il n'est pas aisé d'identifier les tendances regroupant plusieurs pays. Par exemple, les pays nordiques, en particulier scandinaves, sont plutôt vers le pôle SE, tandis que la France et plusieurs pays francophones (Tunisie, Bénin, Sénégal, Burkina-Faso) sont plutôt vers le pôle biomédical, avec l'exception notoire du Maroc, où 2/3 des enseignants ont privilégié le pôle SE. II n'y a pas non plus de clivage entre les pays les moins ou les plus développés sur le plan économique : les pays africains, par exemple, sont dispersés le long de l'échelle de la figure 1. II n'y a pas non plus de tendance très nette corrélée à la religion catholique ou protestante : certes, la Suède et la Finlande, de culture protestante, sont clairement vers le pôle SE, mais la Lituanie, catholique, est aussi située vers ce pôle SE, dont ne sont guère éloignés I'Italie et Malte, en majorité catholiques. La France et la Pologne, où le catholicisme est la religion majoritaire, sont nettement vers le pôle BM. La même diversité s'observe au sein des enseignants de religion orthodoxe : la Roumanie est vers le pôle BM tandis que la Russie ou la Serbie sont plus vers le pôle SE.

D’autres paramètres contrôlés différencient-ils les conceptions des enseignants? 


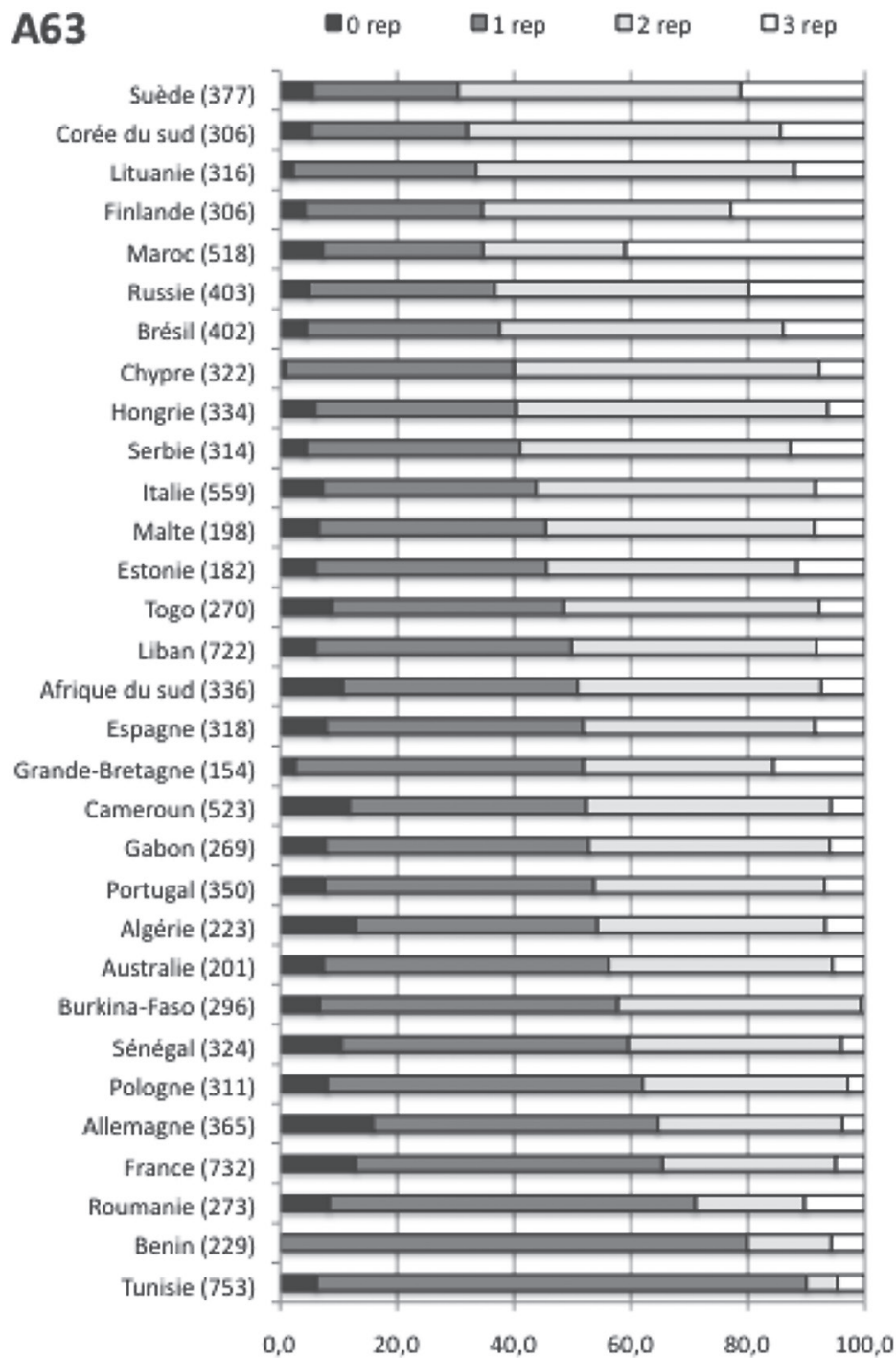

Figure 1 : réponses des 11312 enseignants interrogés à la question A63

Les réponses sont regroupées par pays.

rep = réponse socio-écologique (SE);

0 rep (noir) = aucune réponse SE (les 3 items choisis sont bio-médicaux BM);

1 rep (gris foncé) $=1$ item SE et 2 items BM ;

2 rep (gris clair) $=2$ items SE et 1 item BM ;

3 rep (blanc) $=3$ items SE (aucun item BM) 


\subsection{Différences liées à d'autres paramètres}

Il est possible de regrouper les 11312 enseignants interrogés non plus en fonction de leur nationalité, mais en fonction de chacun des paramètres contrôlés suivants : sexe, âge, catégorie d'enseignants (les six échantillons contrôlés lors du recueil de données), niveau d'instruction (mesuré par le nombre d'années de formation de type universitaire), degré de croyance en Dieu ou de pratique religieuse, religion.

Étant donné le grand nombre d'enseignants, les différences entre ces groupes sont toutes très significatives (tests de Chi2).

Cependant, ces différences peuvent être des biais, si elles ne sont que de simples conséquences des différences entre pays. Prenons l'exemple du sexe. La proportion de femmes enseignantes est très importante dans la plupart des pays, à l'exception des pays africains, où les hommes représentent souvent la moitié ou plus des enseignants interrogés. La différence très significative entre hommes et femmes n'exprime donc peut-être qu'une différence entre pays africains et autres pays. Pour tester cela, nous effectuons une ACPVIO (Analyse en Composantes Principales sur Variables Indépendantes Orthogonales : Sabatier, Lebreton \& Chessel, 1989) qui supprime l'effet pays; nous réalisons ensuite une analyse discriminante (Dolédec \& Chessel, 1989) pour différencier les hommes des femmes, complétée par un test de randomisation de type Monte-Carlo (Romesburg, 1985) attribuant 1000 fois, au hasard, un sexe à chaque enseignant interrogé : les variances observées durant ces 1000 essais sont représentées sous forme d'un histogramme (figure 2) ce qui permet de situer la variance observée (après suppression de l'effet pays) soit hors de cet histogramme : la différence entre hommes et femmes est alors significative même après suppression de l'effet pays; soit au sein de cet histogramme (figure 2) : la différence entre hommes et femmes n'est plus significative; la différence significative initialement observée (avant suppression de l'effet pays) n'était donc que la conséquence de l'inégale répartition hommes/femmes dans les échantillons des différents pays. Réciproquement, nous pouvons supprimer l'effet sexe pour tester si les différences entre pays sont encore significatives, ce qui est le cas (figure 3). Il est donc possible d'en déduire que l'effet sexe n'était qu'une conséquence de l'effet pays, alors que ce dernier est indépendant de l'effet sexe.

Nous avons effectué ces analyses pour chacun des paramètres contrôlés, et nous avons ainsi montré que l'effet de l'âge, celui des religions (figure 4), celui du niveau d'instruction des enseignants et celui des degrés de pratiques religieuses, disparaissent quand on supprime l'effet pays, alors que l'effet pays reste très significatif $(p<0,0001)$ si l'on supprime l'ensemble des effets qui viennent d'être cités (figure 3$)$.

Un seul effet reste significatif après suppression de l'effet pays, différenciant légèrement les six échantillons d'enseignants (figure 5). La figure 6 montre que ces différences, bien que significatives, ne sont pas importantes : les enseignants du primaire sont légèrement moins nombreux à avoir coché deux ou trois items biomédicaux $(48,1 \%$ des enseignants du primaire en fin de formation : PreP; 50,8\% en service : InP) contre, à l'autre extrémité, 55,4\% des enseignants de langue en fin de formation (PreL). 
Genre, supp Pays

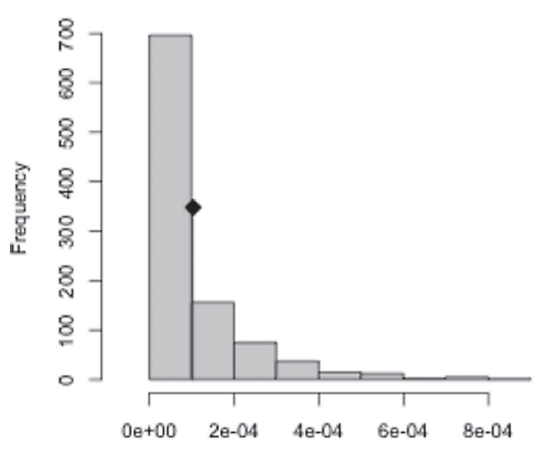

$\operatorname{sim}$

Figure 2 : test de Monte-Carlo sur analyse discriminante testant le facteur sexe sur la variable A63, après ACPVIO supprimant l'effet pays

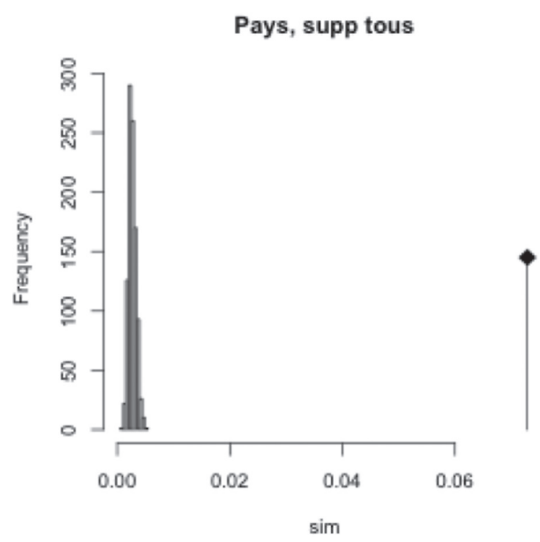

Figure 3 : test de Monte-Carlo sur analyse discriminante testant le facteur pays après une nouvelle ACPVIO

Cette ACPVIO porte sur la variable A63 après suppression de l'effet de tous les facteurs autres que le pays différenciant significativement les réponses des enseignants (six échantillons, sexe, âge, niveau

d'instruction, religion, pratique religieuse).

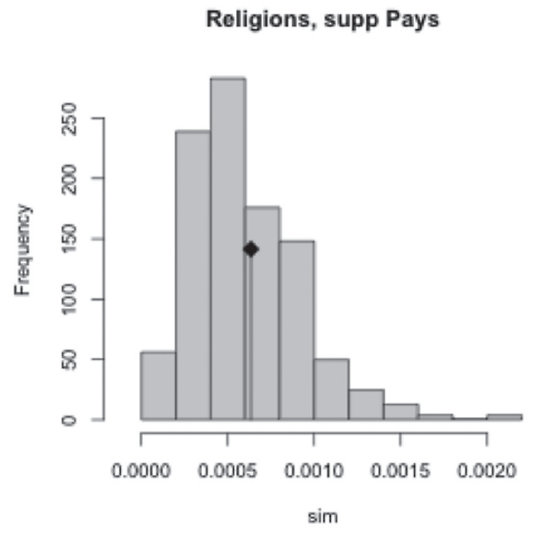

Figure 4 : test de Monte-Carlo sur analyse discriminante testant le facteur religions après ACPVIO supprimant l'effet pays

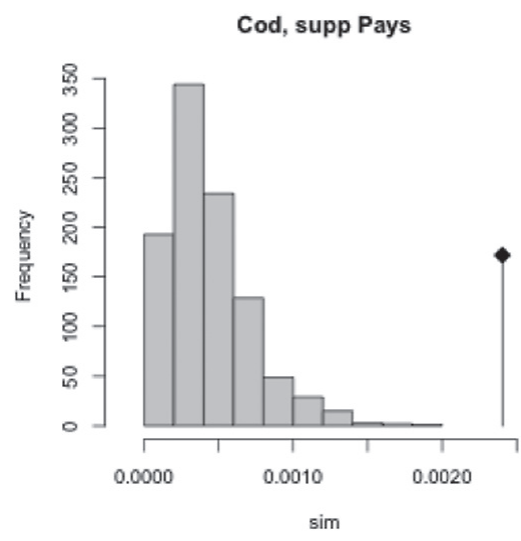

Figure 5 : test de Monte-Carlo sur analyse discriminante testant le facteur "échantillons d'enseignants" après ACPVIO supprimant l'effet pays 


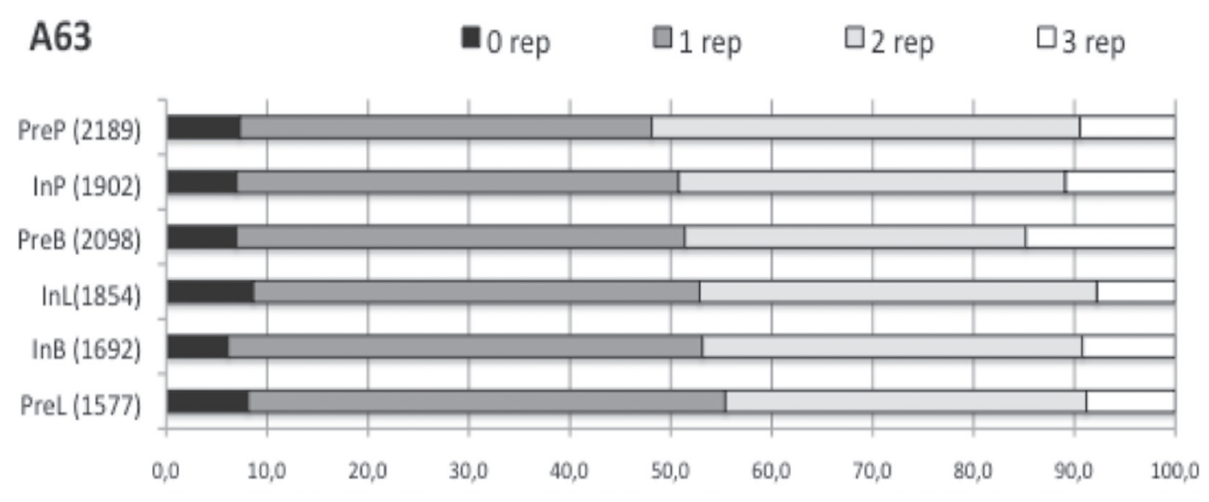

Figure 6 : réponses des 11312 enseignants interrogés à la question A63

Les réponses sont regroupées par catégories d'enseignants. En noir, aucun des 3 items socio-écologiques n'a été coché (donc 3 items biomédicaux cochés). En blanc, l'inverse : 3 items socio-écologiques cochés, et aucun item biomédical.InP : enseignants du primaire en service; PreP : enseignants du primaire en fin de formation; InB : enseignants du secondaire, biologie, en service; PreB : enseignants du secondaire, biologie, en fin de formation; InL : enseignants du secondaire, langue du pays, en service; PreL : enseignants du secondaire, langue du pays, en fin de formation.

\section{Discussion}

Nos résultats montrent que, dans les 31 pays pour lesquels nous disposons de données, les conceptions de la majorité des enseignants interrogés ne sont pas situées vers un seul des deux pôles, biomédical (BM) ou socio-écologique (SE), mais combinent les deux approches. La figure 6 est claire à cet égard : d'une part autour de $50 \%$ des réponses des six catégories d'enseignants expriment une conception biomédicale, et autour de $50 \%$ une conception socio-écologique; d'autre part les réponses où ont été cochés uniquement trois items de l'un de ces deux pôles sont très minoritaires.

Le fait que les enseignants du primaire soient un peu plus ouverts sur la promotion de la santé que leurs collègues du secondaire avait déjà été noté pour les 16 pays analysés dans le rapport final du projet BIOHEAD-Citizen (Carvalho, 2008). Cette différence avait alors été confirmée par l'analyse des manuels scolaires des mêmes pays (Carvalho, Dantas et al., 2008), montrant que la proportion des textes et des images relatifs au modèle biomédical augmente avec l'âge des élèves, en particulier au niveau des plus âgés (16-18 ans), alors que les textes et images relatifs au modèle socio-écologique sont les plus nombreux dans les manuels pour les enfants les plus jeunes (6-9 ans). Il est intéressant de noter que cette tendance reste significative au niveau des conceptions des enseignants interrogés dans 31 pays, tout en n'étant pas très importante (figure 6). Des analyses plus poussées des programmes et manuels scolaires mériteraient d'être mises en œuvre dans les 15 pays non pris en compte dans le rapport de 2008.

Nos résultats montrent aussi des différences importantes entre les 31 pays (figure 1). À partir de données aussi volumineuses, des différences significatives existent aussi 
en fonction des autres paramètres contrôlés (sexe, âge, niveau d'instruction, religion), différences qui ont aussi été notées à partir des données recueillies dans 15 des pays impliqués dans le projet BIOHEAD-Citizen (Berger et al., 2011; Jourdan et al., 2013), sans qu'ait alors été vérifiée la possibilité que ces différences ne soient que des conséquences de l'effet pays. Nous l'avons vérifié sur les données obtenues dans 31 pays, en nous limitant aux réponses à la question A63 (choisir 3 items parmi 6, dont 3 définissent la santé dans ses dimensions biomédicales, et 3 dans ses dimensions socio-écologique/ promotion de la santé). Et nos résultats montrent que chacun de ces effets n'est en fait que la conséquence de l'effet pays, le sexe, l'âge, le niveau d'instruction ou la religion des enseignants étant inégalement répartis d'un pays à un autre. En revanche l'effet pays reste très significatif si l'on supprime les effets liés à ces paramètres contrôlés. Pour répondre à l'hypothèse formulée plus haut sur l'effet possible de la religion déclarée par chaque enseignant, nos résultats montrent donc que la religion est un paramètre des caractéristiques socioculturelles de chaque pays, caractéristiques qui différencient fortement les pays entre eux. Cependant, la religion n'a pas d'effet spécifique : au sein de chaque pays, elle ne différencie pas significativement les conceptions des enseignants interrogés; elle n'a pas, sur ce thème, d'effet indépendant de l'effet pays.

II serait intéressant d'analyser pour chacun des 31 pays les caractéristiques qui peuvent expliquer les conceptions que nous avons mises en évidence. Comme indiqué dans l'introduction du présent article, l'ensemble du projet BIOHEAD-Citizen a tenté d'analyser les conceptions des enseignants en tant que possibles interactions entre leurs connaissances scientifiques (K pour knowledge), leurs valeurs $(\mathrm{V})$ et leurs pratiques sociales (P), selon le modèle KVP (Clément, 2004, 2010, 2016). Les résultats que nous avons présentés sont une nouvelle illustration de ces interactions KVP. En effet, la définition de la santé par ses paramètres biomédicaux (BM : absence de maladie) correspond à des connaissances scientifiques qui sont toujours développées avec succès par des recherches, sont enseignées dans les facultés de médecine et pratiquées par les médecins et autres soignants. Mais, comme nous l'avons présenté au tout début de cet article, l'élargissement de la conception de la santé aux dimensions socio-écologiques (SE) de la promotion de la santé, déjà prôné par l'OMS en 1986, correspond aussi à des connaissances scientifiques qui ont établi le lien entre santé et paramètres sociaux/environnementaux. Ces deux pôles de connaissances BM et SE s'articulent chacun à des pratiques sociales qui peuvent être complémentaires, tout en ne s'exprimant pas dans les mêmes lieux : lieux dédiés à la guérison de maladies pour le pôle BM (cabinets médicaux, hôpitaux, etc.), luttes sociales et environnementales, à dimensions souvent politique, pour le pôle SE (conflits sur les conditions de travail, luttes contre la pauvreté ou encore contre des pollutions, etc.). Or ces deux pôles correspondent aussi à des valeurs, mises en évidence aussi bien par Foucault (1975) que Canguilhem (1979), avec deux conceptions de l'articulation entre science et progrès, ou entre normal et pathologique.

Nos résultats montrent que, pour la grande majorité des enseignants interrogés dans les 31 pays de notre échantillon, ces deux pôles BM et SE sont plus 
complémentaires qu'antagonistes, mais avec des différences très significatives entre pays, qui traduisent des influences socioculturelles nationales qui mériteraient d'être analysées plus en détail.

Des différences plus nettes encore avaient été observées à partir de l'analyse des passages relatifs à la santé dans 71 manuels scolaires de sciences pour le primaire, de biologie pour le secondaire, dans 16 de ces pays (Carvalho, Dantas et al., 2008; figures 7 et 8). La fréquence des images (figure 7) et le nombre de phrases du texte (figure 8) ont été recensés à partir de grilles précises relevant des catégories suivantes: aspects pathologique, curatif ou préventif pour le pôle biomédical (BM); être en bonne santé ("healthy"), fort, sûr de soi ("empowerment"), ou en lien avec des dimensions environnementales ("environmental»), pour le pôle socio-écologique (health promotion, SE). Cet article cite un exemple de phrase relevant de la catégorie " healthy": "Les enfants qui sont en phase de croissance ont besoin de plus de protéines que les adultes", et un autre exemple pour la catégorie "empowerment": "Soyez fort contre les drogues : résistez à la séduction et à la pression de vos proches, défendez vos propres valeurs et prenez des distances par rapport aux modes morbides".



Figure 7 : proportion d'images BM ou SE dans les manuels scolaires de biologie de 16 pays "Biomedical" = modèle biomédical (BM); " Health Promotion" = modèle socio-écologique (SE). Recherche réalisée dans le cadre du projet BIOHEAD-Citizen : figure extraite de Carvalho, Dantas et al. (2008). 




घ Biomedical (\%) $\quad$ Health Promotion (\%)

Figure 8 : proportion de textes BM ou SE dans les manuels scolaires de biologie de 16 pays "Biomedical" = modèle biomédical (BM); "Health Promotion" = modèle socio-écologique (SE). Recherche réalisée dans le cadre du projet BIOHEAD-Citizen : figure extraite de Carvalho, Dantas et al. (2008).

Cette analyse des manuels scolaires met en évidence des différences entre pays plus contrastées encore que celles observées dans les conceptions des enseignants (figure 1). Ainsi en Allemagne 2/3 des textes et 3/4 des images sont classés dans le pôle promotion de la santé, qui est aussi majoritaire dans les pays scandinaves tels que la Finlande, alors qu'en France ou en Pologne $99 \%$ de textes, et respectivement $93 \%$ et $73 \%$ d'images, ne relèvent que du pôle biomédical BM.

Nous avons constaté dans l'introduction de cet article que ces analyses de manuels scolaires semblaient conforter l'opposition proposée par Faure (1997) entre pays de culture protestante ou catholique. Cependant les résultats présentés plus haut sur les conceptions des enseignants ne confirment que partiellement cette opposition. Certes les enseignants suédois ou finlandais, de culture protestante, se situent majoritairement vers le pôle SE, contrairement aux enseignants français ou polonais, de culture catholique et nettement situés vers le pôle BM (figure 1). Mais la corrélation entre les conceptions d'enseignants et celles exprimées par les manuels scolaires de leur pays n'est pas toujours significative. Par exemple les manuels scolaires sont largement SE en Allemagne, alors que les enseignants sont plutôt vers le pôle BM (à noter qu'en Bavière, il y avait dans notre échantillon autant d'enseignants catholiques que protestants). Par ailleurs, nos analyses ont montré que la religion ne différencie pas significativement les conceptions des enseignants dès que l'on supprime l'effet pays (ce dernier effet restant très significatif si l'on supprime l'effet religion). Cet "effet pays" recouvre des dimensions socioculturelles multiples, qui ont intégré l'histoire religieuse du pays. L'ensemble de ces résultats n'invalide donc pas les analyses 
historiques de Faure (1997), mais montre (1) que la religion n'a plus aujourd'hui le caractère clivant qu'elle a pu avoir et (2) que les caractéristiques socioculturelles d'un pays, incluant son histoire, semblent avoir plus de poids sur les programmes et manuels scolaires que sur les conceptions des enseignants qui opposent moins, d'un pays à un autre, les pôles BM et SE que ne le font les manuels scolaires.

Nos résultats montrent qu'il n'y a pas d'opposition claire entre les pays les plus développés (sur le plan économique) et ceux qui le sont le moins, ni de différences liées au continent ou au climat. Ils suggèrent en revanche une possible influence du système éducatif. Ainsi le système scolaire du Mozambique est proche de celui du Portugal : leurs manuels scolaires respectifs expriment le même type d'équilibre entre pôles BM et SE (avec légèrement plus d'images SE au Mozambique). On retrouve ce type d'équilibre dans les conceptions des enseignants portugais (il est dommage que l'équipe du Mozambique n'ait pas réalisé l'enquête auprès d'enseignants de leur pays). Cet équilibre vient de la volonté du Portugal de développer le pôle SE dans son système scolaire (Carvalho, 2012). Plus nettement encore, les manuels tunisiens et algériens (analysés après la publication de Carvalho, Dantas et al., 2008) expriment une large prédominance du pôle biomédical, qui caractérise aussi les manuels français ainsi que les conceptions des enseignants de ces trois pays (figure 1), conceptions biomédicales que l'on retrouve majoritaires dans les pays africains francophones: au Bénin (figure 1), mais aussi au Sénégal (figures 1, 7, 8) et au Burkina Faso (figure 1). Mais le cas du Maroc illustre que ce n'est pas si simple. Au Maroc, 2/3 des enseignants ont coché des items plutôt SE (figure 1), ce qui correspond en partie au contenu des manuels scolaires analysés dans le cadre du projet BIOHEAD-Citizen (figures 7 et 8) : près de $50 \%$ des textes, et $30 \%$ des images, relevaient de la promotion de la santé (contre $1 \%$ et $7 \%$ en France). Cependant, c'est surtout à l'école élémentaire qu'est présente la perspective SE (bonne santé, habitudes saines), la perspective BM devenant ensuite largement dominante jusqu'au bac. L'élargissement des conceptions sur la santé, promu par l'OMS en 1986, n'est donc encore que partiel au Maroc, où il est cependant plus important qu'en France, d'une part sur les programmes et manuels scolaires et plus encore sur les conceptions des enseignants. Il s'agit, au Maroc, d'une volonté politique dont a par exemple témoigné la participation de trois ministres marocains (Éducation et Recherche; Santé; Développement social de la famille et solidarité) au colloque "Promotion de la santé en milieu scolaire" (Rabat, 2-6 avril 2007) pour prendre connaissance des résultats de la recherche BIOHEADCitizen sur ce thème au Maroc (Selmaoui et al., 2007).

Le délai de transposition didactique (DTD : Quessada \& Clément, 2007; Clément, 2014b) mesure le temps qui sépare une nouvelle conception scientifique de son introduction dans les programmes (DTDp) et/ou dans les manuels scolaires (DTDm) d'un pays, ainsi que dans les conceptions des enseignants de ce pays (DTDc). En ce qui concerne la santé, on peut dater de 1986 l'élargissement par l'OMS de la conception biomédicale aux dimensions socio-écologiques de la promotion de la santé. II ne s'agit pas de remplacer le pôle BM par le pôle SE, mais d'introduire les deux pôles dans 
l'éducation à la santé. Les analyses des manuels scolaires effectuées en 2006-2007 (Carvalho, Dantas et al., 2008) ont montré que ce DTD différait beaucoup d'un pays à un autre. Cette nouvelle définition avait, par exemple, déjà été intégrée au Maroc en 2006 (DTD < 20 ans) alors que ce n'était pas encore le cas en France (DTD $>20$ ans).

Comparée à d'autres pays européens, la France a une longue et tenace conception biomédicale de la santé (Jourdan et al., 2013; Pommier \& Jourdan, 2007). Son système scolaire a longtemps couplé l'approche de la santé à l'étude du corps humain, et ceci dès l'école maternelle (voir par exemple Bornancin, Bornancin \& Moulart, 1997).

II serait intéressant d'approfondir l'analyse de cette tradition biomédicale de la santé en France, et de tenter de comprendre pourquoi cet élargissement de la définition de la santé met (ou a mis) tant de temps à s'y mettre en place. La question est complexe et inclut des dimensions politiques qui illustrent des interactions KVP. Ainsi, la comparaison des programmes des candidats à l'élection présidentielle de 2017 met en évidence des oppositions claires quant à leurs conceptions sur la santé publique (Santi \& Brajeul, 2017). Par exemple certains candidats dénoncent plus clairement que d'autres le poids des lobbies pharmaceutiques dans la formation et le recyclage des médecins, préconisent plus nettement des mesures pour lutter contre les dégâts du tabac, de l'alcool, des pesticides et autres pollutions, ou encore pour mieux informer de la qualité nutritionnelle des aliments afin de prévenir l'obésité : autant de domaines qui peuvent s'appuyer sur des études scientifiques solides (K), mais où les pratiques $(\mathrm{P})$ divergeront en fonction de valeurs $(\mathrm{V})$ différentes.

En France, l'éducation à la santé mérite d'être développée en lien avec l'éducation à l'environnement et au développement durable, ainsi qu'en lien avec l'éducation à la citoyenneté. II ne s'agit pas bien sûr de ne plus enseigner le corps humain, ni les pathologies et la prévention, mais d'intégrer ces dimensions biomédicales essentielles dans une perspective positive et holistique de la santé. La formation des enseignants devrait comprendre plus clairement qu'aujourd'hui cette ouverture vers les dimensions socio-écologiques de la santé.

Terminons sur une note optimiste en soulignant à nouveau que, en moyenne pour les 11312 enseignants interrogés dans 31 pays, les deux conceptions BM et SE de la santé sont présentes à part égale (figure 6) : elles sont effectivement complémentaires. Au niveau international, la conception de la santé, initialement réduite à sa dimension biomédicale, s'est déjà bien élargie dans nombre de pays, intégrant aussi désormais les dimensions socio-écologiques de la promotion de la santé. 


\section{Bibliographie}

ANTONOVSKY A. (1979). Health, Stress and Coping. San Francisco : Jossey-Bass Publishers.

ANTONOVSKY A. (1996). The salutogenic model as a theory to guide health promotion. Health Promot International, vol. 11, $\mathrm{n}^{0} 1, \mathrm{p} .11-18$.

BERGER D., BERNARD S., WAFO F., HRAIRI S., BALCOU M. \& CARVALHO G.S. (2011). Éducation à la santé et à la sexualité : qu'en pensent les enseignants? Étude comparative dans 15 pays. Carrefour de l'éducation, nº 32, p. 75-97.

BORNANCIN B., BORNANCIN M. \& MOULART D. (1997). À la découverte du corps et de la santé. Apprendre à l'école maternelle. Nice : Z' éditions.

BRISSET C. (1984). La santé dans le tiers monde. Paris : La Découverte/Le Monde.

CANGUILHEM G. (1979). Le normal et le pathologique. Paris : Presses universitaires de France.

CARVALHO G. S. (2006). Criação de ambientes favoráveis para a promoção de estilos de vida saudáveis. In B. Pereira \& G. S. Carvalho (éd.), Actividade física, saúde e lazer: A infância e estilos de vida saudáveis, Lisbonne : Lidel, p. 19-37.

CARVALHO G. S. (2008). Health Education topic: Objectives, methodologies, main achievements and implication. In G. Carvalho, P. Clément, F. Bogner \& S. Caravita, BIOHEAD-Citizen: Biology, Health and Environmental Education for better Citizenship, Final Report. Bruxelles : FP6, Priority 7, Project $\mathrm{n}^{\circ}$ CITC-CT-2004-506015, p. 10-27.

CARVALHO G. S. (2012). Health Education in Portuguese Schools: The Contribution of Health and Education Sectors. In N. Taylor, M. Littledyke, F. Quinn \& R. K. Coll (éd.), Health education in context: An International Perspective on Health Education in Schools and Local Communities, Rotterdam : Sense Publishers, p. 37-46.

CARVALHO G.S. \& BERGER D. (2012). School Health Education Nowdays: Challenges and Trends. In D. Jorde and J. Dillon (eds.) Science Education Research and Practice in Europe: Retrospective and Prospective. Rotterdam : Sense Publishers. p. 309-340.

CARVALHO G. S., CLÉMENT P., BOGNER F. \& CARAVITA S. (2008). BIOHEAD-Citizen: Biology, Health and Environmental Education for better Citizenship, Final Report. Bruxelles : FP6, Priority 7, Project $n^{\circ}$ CITC-CT-2004-506015

CARVALHO G. S., DANTAS C., RAUMA A. L., LUZI D., RUGGIERI R., BOGNER F., GEIER C., CAUSSIDIER C., BERGER D. \& CLÉMENT P. (2008). Comparing health 
education approaches in textbooks of sixteen countries. Science Education International, vol. 19, nº 2, p. 133-146. En ligne : <http://www.icaseonline.net/ sei/june2008/19-2-june-2008-133_146.pdf >.

CASTÉRA J. \& CLÉMENT P. (2014). Teachers' conceptions about genetic determinism of human behaviour: a survey in 23 Countries. Science and Education, vol. 23, $\mathrm{n}^{0} 2$, p. 417-443. En ligne : < http://hal.archives-ouvertes.fr/hal-01024280>.

CHRAPPÁN M. (2012). The possibility of health education in an education-based society: The case of Hungary. In N. Taylor, M. Littledyke, F. Quinn \& R. K. Coll (éd.), Health education in context: An International Perspective on Health Education in Schools and Local Communities, Rotterdam : Sense Publishers, p. 47-56.

CLÉMENT P. (2004). Science et idéologie : exemples en didactique et épistémologie de la biologie. Actes du Colloque Sciences, médias et société. ENS-LSH, p. 53-69. En ligne : $<$ http://sciences-medias.ens-lyon.fr/article.php3?id article $=58>$.

CLÉMENT P. (2010). Conceptions, représentations sociales et modèle KVP. Skholê, $\mathrm{n}^{0} 16$, p. 55-70. En ligne : <http://hal.archives-ouvertes.fr/hal-01024972>.

CLÉMENT P. (2014a). Les conceptions créationnistes d'enseignants dans 30 pays. Varient-elles en fonction de leur religion? Éducation et sociétés, vol. 33, $\mathrm{n}^{\circ} 1$, p. 113-136.

CLÉMENT P. (2014b). Le délai de transposition didactique dans les livres du professeur. Quelques exemples en SVT classe de $3^{\mathrm{e}}$. Skholê, vol. 18, n ${ }^{0} 1$, p. 109-120. En ligne : <http://hal.archives-ouvertes.fr/hal-01024279>.

CLÉMENT P. (2016). 0 modelo KVP: Interações entre Conhecimentos, Valores e Práticas sociais. In E.T. Oliveira Boff, M.C. Pasera-de-Araùjo \& G.S. Carvalho, Conhecimentos, valores e práticas na educação em saúde, ljui (Brésil): Éd. Unijui, Coleção nas ciências, , p. 13-30.

CLÉMENT P. \& CARVALHO G. (2007). Biology, Health and Environmental Education for better Citizenship: teachers' conceptions and textbook analysis in 19 countries. Proceedings WCCES XIII (World Council of Comparative Education Societies), Sarajevo, Cédérom, $15 \mathrm{p}$.

CONRADIE F. (2012). Medical research in South Africa: Education and ethics. In N. Taylor, M. Littledyke, F. Quinn \& R. K. Coll (éd.), Health education in context: An International Perspective on Health Education in Schools and Local Communities, Rotterdam : Sense Publishers, p. 99-106.

DOLÉDEC S. \& CHESSEL D. (1989). Rythmes saisonniers et composantes stationnelles en milieu aquatique. II : Prise en compte et élimination d'effets dans un tableau faunistique. Acta OEcologica, CEcologia Generalis, $n^{0} 10$, p. 207-232. 
EWLES L. \& SIMNETT I. (1999). Promoting health. A practical guide. Londres : Baillière Tindall.

FAURE 0. (1997). Les stratégies sanitaires. In M.D. Grmek, Histoire de la pensée médicale en Occident, vol. 2 : De la Renaissance aux Lumières, p. 279-296.

FOUCAULT M. (1975). Surveiller et punir. Paris : Gallimard.

FUDDAH H. (2014). Health Education at schools in Lebanon. Critical analyses of curriculum, textbooks, focus groups and classroom sequences in a Lebanese public school and a Palestinian school. Thèse de doctorat, Beyrouth : université libanaise.

FUDDAH H., KHALIL I. \& CLÉMENT P. (2015). Health Knowledge, Attitudes, Beliefs and Practices revealed from Focus Group Discussions on Tobacco Smoking among Lebanese and Palestinian Schoolchildren in Lebanon. International Journal of Science and Research (IJSR), n ${ }^{\circ} 4-9$ septembre 2015.

HERZLICH C. (1992). La santé et les préoccupations de santé aujourd'hui. In L'homme et la santé, Paris : Seuil, p. 91-95.

HERZLICH C. (1995). De la médecine triomphante à l'ère des doutes. La Recherche, supplément au n² 281, La santé et ses métamorphoses, p. 32-34.

JOURDAN D., PIRONOM J., BERGER D. \& CARVALHO G.S. (2013). Factors influencing teachers' views of health and health education: A study in 15 countries. Health Education Journal, vol. 72, nº 6, p. 660-672.

GEORGE S. (1978). Comment meurt l'autre moitié du monde. Paris : Robert Laffont.

GVOZDEVA M. \& KIRILINA V. (2012). Education for healthy lifestyles in the European north of Russia: Developments and dilemmas. In N. Taylor, M. Littledyke, F. Quinn \& R. K. Coll (éd.), Health education in context: An International Perspective on Health Education in Schools and Local Communities, Rotterdam : Sense Publishers, p. 67-76.

KANJ M. (2012). Health education through extra curricular activities in Lebanon: Process and challenges. In N. Taylor, M. Littledyke, F. Quinn \& R. K. Coll (éd.), Health education in context: An International Perspective on Health Education in Schools and Local Communities, Rotterdam : Sense Publishers, p. 27-36.

KATZ J., PEBERDY A. \& DOUGLAS J. (2000). Promoting Health: Knowledge and Practice. Basingstoke: Palgrave.

MARTINS L., EL-HANI C.N. \& CARVALHO G.S. (2016). A saúde nos livros didáticos brasileiros de biologia: que ensino propomos? In M.C. Pansera, E. Boff \& G.S. Carvalho (éd.) Conhecimentos, Valores e práticas na Educação em Saúde, Ijuí : Éd. Unijuí, p. 133-156. 
MOATTI J.P., DAB W., POLLAK M. et al. (1990). Les attitudes et comportements des Français face au sida. La Recherche, $\mathrm{n}^{\circ}$ 223, p. 888-895.

NAIDOO J. \& WILLS J. (1994). Health Promotion. Foundations for Practice. London : Baillière Tindall.

OMS (ORGANISATION MONDIALE DE LA SANTÉ) (1946). Preamble to the Constitution of the World Health Organization. New York, 19 Juin-22 juillet 1946.

OMS (ORGANISATION MONDIALE DE LA SANTÉ) (1986). Promotion de la Santé, Charte d'Ottawa. En ligne : < http://www.euro.who.int/_data/assets/pdf file/0003/129675/0ttawa_Charter_F.pdf $>$.

PLATJE J. \& SŁODCZYK K. (2012). The role of health education in preventing diseases: A case study from Poland. In N. Taylor, M. Littledyke, F. Quinn \& R. K. Coll (éd.), Health education in context: An International Perspective on Health Education in Schools and Local Communities, Rotterdam : Sense Publishers, p. 77-86.

POMMIER J., GUÉVEL M.-R. \& JOURDAN D. (2010). Evaluation of health promotion in schools: a realistic evaluation approach using mixed methods. BMC Public Health. 10: 43. En ligne : <https://www.ncbi.nlm.nih.gov/pmc/articles/ PMC2824736/>.

POMMIER J. \& JOURDAN D. (2007). La santé à l'école dans les pays européens. Paris : Sudel.

QUESSADA M.P. \& CLÉMENT P. (2007). An epistemological approach to French curricula on human origin during the 19th \& 20th centuries. Science and Education, vol. 16, no 9-10, p. 991-1006.

R_DEVELOPMENT_CORE_TEAM. (2006). R: A language and environment for statistical computing, Vienne : R Foundation for Statistical Computing.

ROMESBURG H-C (1985). Exploring, confirming, and randomization tests. Computers and Geosciences, vol. 1, $\mathrm{n}^{0} 1$, p. 19-37.

SABATIER R., LEBRETON J.D. \& CHESSEL D. (1989). Principal Component Analysis with Instrumental Variables as a Tool for Modelling Composition Data. In R. Coppi \& S. Bolasco (éd.) Multiway Data Analysis, Amsterdam : Elsevier Science Publishers, p. 341-352.

SANTI P. \& BRAJEUL C. (2017). Santé publique. Le grand écart des programmes. Le Monde science et médecine, 12 avril 2017, p. 4-5.

SELMAOUI S., KHZAMI S., AGORRAM B. \& ELABBOUDI T. (2007). L'éducation à la santé à l'école : Quelles approches éducatives? Actes du colloque Promotion de la santé en milieu scolaire, Rabat (2-6 avril). Power point dans les Actes, CD-Rom de l'AESVT, Rabat. 
TAYLOR N., QUINN F., LITTLEDIKE M. \& COLL R. K. (2012). Health education in context: An international perspective on health education in schools and local communities. Rotterdam : Sense Publishers.

YAHYA N. A. \& ALI N. A. M. (2012). Malaysian traditional knowledge and herbal gardens: Informal education on plant resources for health. In N. Taylor, M. Littledyke, F. Quinn \& R. K. Coll (éd.), Health education in context: An International Perspective on Health Education in Schools and Local Communities, Rotterdam : Sense Publishers, p. 191-198.

ZEYER A. \& KYBURZ-GRABER R. (éd.) (2012). Science/Environment/Health. Towards a renewal pedagogy for science education. Springer. 